\title{
Artigos científicos publicados em acesso aberto pelos docentes dos programas de pós-graduação stricto sensu no Setor de Ciências Sociais Aplicadas da UfFR
}

\section{Scientific articles published in open access by the professors of the stricto sensu graduate programs in the Social Sciences Sector Applied at UFPR}

\author{
Karolayne Costa Rodrigues de Lima ${ }^{1}$, Ricardo Mendes Junior ${ }^{2}$, José Simão de Paula Pinto ${ }^{3}$ \\ ${ }^{1}$ Universidade Federal Paraná, Curitiba, Paraná, Brasil. ORCID: http://orcid.org/0000-0002-6311-8482 \\ 2 Universidade Federal do Paraná, Curitiba, Paraná, Brasil. ORCID: https://orcid.org/0000-0003-4947-0364 \\ ${ }^{3}$ Universidade Federal do Paraná, Curitiba, Paraná, Brasil. ORCID: https://orcid.org/0000-0002-5023-437X
}

Autor para correspondência/Mail to: Karolayne Costa Rodrigues de Lima, karoll@ufpr.br

\begin{abstract}
Resumo
Introdução: apresenta o contexto da comunicação científica e os movimentos de acesso aberto à informação focando na criação e no desenvolvimento das formas de publicação: acesso aberto ou acesso restrito. Contextualiza os sistemas de publicação científica e a disponibilidade e o acesso aos artigos científicos produzidos com recursos públicos. Visa responder qual é a forma de publicação acadêmica adotada pelos docentes dos programas de pós-graduação stricto sensu do Setor de Ciências Sociais Aplicadas da Universidade Federal do Paraná para a comunicação dos resultados de pesquisa Objetivo: apresenta como objetivo geral identificar por meio da plataforma Lattes e enquanto docente do quadro permanente dos programas de pós-graduação stricto sensu da Universidade Federal do Paraná qual é a forma de publicação adotada para a comunicação dos resultados de pesquisa. Metodologia: o método utilizado para identificação do tipo de sistema de publicação é a aplicação de um algoritmo de coleta e classificação de artigos científicos. Conclusão: os resultados indicam que $54 \%$ dos docentes do setor publicam seus artigos em acesso aberto.
\end{abstract}

Palavras-chave: Acesso aberto; Comunicação científica; Produção científica - Universidade Federal do Paraná.

\begin{abstract}
Introduction: it presents the context of scientific communication and movements of open access to scientific information focusing on the creation and development of forms of publication: open access or restricted access. It contextualizes the systems of scientific publication and the availability and access to scientific articles produced with public resources. It aims to answer the form of academic publication adopted by professors of the stricto sensu postgraduate programs of the Applied Social Sciences Sector of the Universidade Federal do Paraná for the communication of research results. Objective: it presents with general objective to identify through the platform Lattes and as professor of the permanent framework of the graduate stricto sensu programs of UFPR what is the form of the publication adopted for the communication of the results of research. Method: the method used to identify the form of publication system is the application of an algorithm for the collection and classification of scientific articles Conclusion: the results indicate that $54 \%$ of professor in the sector publish their articles in open access..
\end{abstract}

Keywords: Open access; Scientific communication; Scientific production - Universidade Federal do Paraná.

\section{INTRODUÇÃO}

Dos primórdios da comunicação científica até os dias de hoje, o surgimento da internet e o desenvolvimento das Tecnologias de Informação e Comunicação (TIC) propiciam à sociedade o acesso às publicações científicas. Para os pesquisadores, proporcionou a visibilidade de suas pesquisas, a afirmação de seu nome entre os pares e possibilidades ilimitadas de colaboração e de exploração de novos aspectos do conhecimento.

Se antes a comunicação da ciência era lenta e não atingia toda a comunidade acadêmica, hoje o mundo científico parece quase não ter barreiras, pois o acesso aos relatos de pesquisa sistematizados em periódicos é quase instantâneo devido aos sistemas de publicação eletrônica. O "quase", neste contexto, representa a crítica sobre o acesso restrito aos artigos científicos publicados em periódicos fechados (acesso mediante assinatura).

Os modelos de negócios adotados pelas grandes editoras de conteúdo científico conflitam com o próprio sentido da ciência em si. Ora, se um dos propósitos da ciência é explicar a natureza e, assim, indiretamente, servir à sociedade (Moura, 2014), então por que a comunicação da ciência, que é formalizada por meio da publicação de um artigo científico, não é acessível para todos de forma geral?

A maioria dos artigos científicos hoje são acessíveis apenas por meio da assinatura de bases de dados e/ou de periódicos. Essa assinatura é geralmente custeada pelas bibliotecas das instituições de ensino superior ou institutos/centros de pesquisa, ou então de maneira individual pelos pesquisadores. 
A considerar que os artigos científicos são produzidos em ampla medida por pesquisadores/docentes, discentes das universidades e instituições correlatas, é fato que existe investimento de recurso público envolvido na publicação de artigos científicos, subsidiados por meio de recursos para desenvolvimento, manutenção e suporte de projetos e grupos de pesquisa, bolsas de natureza acadêmicas e ou por meio de proventos (no caso dos docentes).

No Brasil, existem hoje 68 universidades federais (INEP, 2018), com quadro total de docentes permanentes de 80.772 (CAPES, 2018). Considerando esses dados, faltam estudos que apontem como está sistematiza a publicação científica brasileira das instituições de ensino superior. A crítica do senso comum é a de que parte da sociedade não compreende o trabalho dos pesquisadores e docentes, pois a comunicação das pesquisas na forma de artigo científico, em sua maioria ou em sua relevância, não está acessível de forma aberta para todos.

A questão da identificação da produção científica é realizada por meio das declarações de publicação dos pesquisadores/docentes informados na Plataforma Lattes, no Sucupira e/ou nos bancos de dados do CNPq. Todavia, existe uma lacuna a respeito da disponibilidade e do acesso aos artigos científicos produzidos pelos docentes nas instituições de ensino superior. Dessa forma, a questão de pesquisa do presente estudo é responder qual é o tipo de sistema de publicação acadêmica adotado pelos docentes dos programas de pós-graduação stricto sensu do Setor de Ciências Sociais Aplicadas da Universidade Federal do Paraná para a comunicação dos resultados de pesquisa.

Nesse sentido, o objetivo geral desta pesquisa é identificar por meio da Plataforma Lattes e enquanto docente do quadro ativo da UFPR, qual é a forma de publicação adotada para a comunicação dos resultados de pesquisa. Para atingir o objetivo proposto, foram selecionados os seguintes objetivos específicos: a) levantar a quantidade de artigos científicos declarados pelos docentes na Plataforma Lattes; b) identificar quantos artigos científicos apresentam o identificador persistente Document Object Identify (DOI) atribuído; e c) classificar a forma de publicação adotada pelos docentes do Setor de Ciências Sociais Aplicadas da UFPR.

A pesquisa justifica-se devido à importância de mensurar o quantitativo dos artigos científicos produzidos pelos docentes nas universidades e constitui-se de fator relevante para a avaliação acadêmica tanto dos programas de pós-graduação, ranking universitário e fomento a novos projetos de pesquisa.

Dentro de um contexto social, a produção científica pode ser compreendida como o retorno à sociedade às necessidades requeridas por estas como uma justificativa à sua existência e manutenção. A identificação, a reunião, a disponibilidade e o acesso à produção das universidades podem promover também a transparência na aplicação de recursos públicos e privados e promover a popularização da ciência, ainda que de forma geral.

A identificação da forma de publicação adotada pelos docentes pode ser compreendida como o início de um trabalho em mapear e compreender o comportamento institucional dos docentes quanto sobre a adesão ao acesso aberto à literatura científica e também explicitar se essa produção está ou não disponível para a própria instituição fomentadora do trabalho docente.

\section{REFERENCIAL TEÓRICO}

Nos primórdios da ciência, a produção científica era centrada em folhetins, jornais diários ou, mais especificamente, na troca de cartas entre os pesquisadores, até que a invenção da imprensa possibilitou mudanças profundas no formato da comunicação científica, a resultar no surgimento dos primeiros periódicos científicos especializados (Freitas, 2006). Desde então, a forma de comunicar a ciência sofreu diversas mudanças e especializações de maneira a fortalecer e propiciar a colaboração entre pesquisas, entre autores e o desenvolvimento de novos métodos e tecnologias.

Seguindo nesse contexto histórico, após séculos de publicação científica, o modelo até então vigente enfrentava uma série de problemas como a “(...) dispersão da informação, atraso na avaliação das submissões, atrasos nas publicações e alto preço das assinaturas dos periódicos" Weitzel (2006, p. 87). Nesse sentido, o avanço constante no desenvolvimento das tecnologias de informação e comunicação possibilitou uma solução para a melhoria dos processos de fazer e comunicar ciência.

Nesse cenário, a internet aparece como a protagonista da transição entre as publicações impressas e o novo formato eletrônico, bem como para os movimentos de acesso aberto à informação científica (Harnard, 2005; Marcondes \& Sayão, 2001).

De acordo com Mueller (2006), o surgimento dos primeiros periódicos eletrônicos e a transição do suporte informacional de impresso para eletrônico não alterou as questões relativas à forma de acesso e à forma de aquisição do conteúdo científico. Os grandes periódicos indexados e mantidos pelas tradicionais editoras comerciais continuaram a ser mandatários quanto ao direito autoral das pesquisas publicadas, mantendo sua política majoritária e de restrição nas questões de acesso e reuso da informação científica.

A mudança nesse cenário viria logo em seguida com as primeiras manifestações que culminaram nos movimentos de acesso aberto à informação científica. O contexto de ebulição desse movimento ocorreu em grande medida a 
partir da dificuldade de bibliotecas, instituições de ensino e de pesquisadores em ter acesso aos artigos científicos devido ao alto custo de manutenção das assinaturas de periódicos e bases de dados, sendo que o movimento só foi possível devido à existência da internet (Harnard, 2005).

Em contrapartida às dificuldades, o movimento do software livre proporcionou um ambiente favorável para o desenvolvimento dos primeiros sistemas de armazenamento e gestão de conteúdo científicos - repositórios digitais, sistema de publicação de periódicos e conferências abertas com licenças abertas do tipo GNU ou Creative Commons.

Considerando então as possibilidades de alargamento de alcance e visibilidade proporcionadas pela internet e as TIC relacionadas, a década de 70 nos Estados Unidos trouxe consigo o movimento de software livre liderado por Stallman (2004) cuja filosofia preconiza a cultura do compartilhamento, divulgação e reuso de software livre por meio da criação e aplicação de licenças abertas. Essas iniciativas deram origem aos movimentos Open Source Initiative, Free Software Foundation (Setenareski, 2013).

A partir da perspectiva desse avanço, a década de 90 refletiu uma esperança de reestruturação nas práticas de publicação científica através de um sistema alternativo para publicação que possibilitasse ao autor conferir ao seu trabalho acesso irrestrito e gratuito.

Como exemplo dessa reestruturação, em 1991, no Los Alamos National Laboratory, o pesquisador Paul Ginparg lançou um repositório denominado ArXiv, por meio do qual tornou disponível em acesso aberto publicações científicas desenvolvidas na área de física. Segundo a Open Archives Initiative (2007), esse repositório foi precursor ao movimento de acesso aberto.

De maneira a fortalecer os primeiros passos para uma ciência aberta, em 1999 ocorreu a Conferência de Santa Fé nos Estados Unidos que definiu uma série de princípios básicos do acesso aberto à informação científica e formalizou a Open Archives Initiative (OAI) (Silva \& Alcará, 2008).

O Brasil acompanhou as iniciativas mundiais do movimento lançando em 1998 o primeiro portal de periódicos científicos em acesso aberto, o Scientific Eletronic Library Online (SciELO), oferecendo acesso gratuito a cerca de 200 periódicos em português e espanhol. O Instituto Brasileiro de Informação em Ciência e Tecnologia (IBICT), unidade de pesquisa do Ministério da Ciência e Tecnologia e, portanto, com posicionamento oficial, aderiu às iniciativas mundiais ao lançar o Manifesto Brasileiro de Apoio ao Livre Acesso à Informação Científica em 2005. A partir do Manifesto, o IBICT elaborou e coordenou diversos projetos e ações para promover o acesso aberto a produções acadêmicas, como por exemplo, a Biblioteca Digital de Teses e Dissertações (BDTD) com o software TEDE, o portal de repositórios e periódicos de acesso livre (OAIS.BR) (uma adaptação e tradução do software Open Journal System (OJS), da Open Knowledge Project e, a iniciativa mais recente, o Plano de Dados Abertos.

Ocorreram diversas outras conferências e eventos em diversos países para consolidação de um movimento de ciência aberta. Um dos eventos mais significativos foi a Budapest Open Access Initiative (BOAI), realizada em fevereiro de 2002, que teve por propósito analisar e fortalecer as demais iniciativas em acesso aberto, a promoção da interoperabilidade entre repositórios e bibliotecas digitais por meio do protocolo automático de coleta Open Archives Initiative - Protocol for Metadata Harvesting - OAI-PMH) e recomendar duas estratégias de publicação: o autoarquivamento (green road ou via verde) e o acesso aberto aos periódicos (golden road ou via dourada) (Silva \& Alcará, 2008).

Segundo Costa (2006), a via verde preconiza o autoarquivamento em repositórios digitais pelo pesquisador de artigos científicos já publicados em periódicos mediante aceite do veículo de publicação. A via dourada é a opção do pesquisador para publicar em periódicos de acesso aberto.

A falta de estudos sobre o comportamento dos pesquisadores e a forma de publicação científica impossibilita a realização de análises sobre o cenário brasileiro quanto à publicação de artigos científicos em acesso aberto, seja pela via verde ou via dourada.

Neste cenário, observa-se desde 1998 o crescimento exponencial de títulos de periódicos em acesso aberto no Brasil. Conforme levantamento realizado no OpenDOAJ , o Brasil possui 1.277 títulos de periódicos cadastrados como acesso aberto.

O dado acima é insuficiente para compreender o comportamento de publicação científica dos pesquisadores no Brasil. No entanto, fica evidente o destaque brasileiro quanto ao volume de publicações. Nesse sentido, a proposta desta pesquisa em identificar qual é o tipo de sistema de comunicação científica adotado pelos pesquisadores da universidade visa contribuir parcialmente na análise do cenário na Universidade Federal do Paraná quanto à produção docente do Setor de Ciências Sociais Aplicadas na pós-graduação.

\section{Caracterização da amostra}

O Setor de Ciências Sociais Aplicadas da Universidade Federal do Paraná é composto por quatro departamentos que contam com um programa de pós-graduação stricto sensu, a saber: Departamento de Administração Pública e Aplicada, Departamento de Contabilidade, Departamento de Ciência e Gestão da Informação e, por fim, 
Departamento de Economia (Universidade Federal do Paraná, 2018). Este último possui dois programas, sendo eles: programa de pós-graduação em Políticas Públicas e Desenvolvimento Econômico, sendo que este último oferece curso de mestrado acadêmico e também o profissional, conforme Quadro 1:

\begin{tabular}{|l|c|l|c|}
\hline Departamento & $\begin{array}{c}\text { Quantidade de } \\
\text { programas stricto sensu }\end{array}$ & Níveis & $\begin{array}{l}\text { Quantidade } \\
\text { de docentes }\end{array}$ \\
\hline $\begin{array}{l}\text { Administração Pública } \\
\text { e Aplicada }\end{array}$ & 1 & $\begin{array}{l}\text { Mestrado acadêmico; } \\
\text { Doutorado acadêmico; } \\
\text { Pós-doutorado. }\end{array}$ & 23 \\
\hline Contabilidade & 1 & $\begin{array}{l}\text { Mestrado acadêmico; } \\
\text { Doutorado acadêmico; } \\
\text { Pós-doutorado. }\end{array}$ & 13 \\
\hline Economia & 3 & $\begin{array}{l}\text { Mestrado profissional; } \\
\text { Mestrado acadêmico; } \\
\text { Doutorado. }\end{array}$ & 43 \\
\hline Ciência e Gestão da Informação & 1 & $\begin{array}{l}\text { Mestrado acadêmico; } \\
\text { Doutorado acadêmico. }\end{array}$ & 12 \\
\hline
\end{tabular}

Quadro 1. Panorama da pós-graduação stricto sensu no setor de ciências sociais aplicadas. Fonte: Dados da pesquisa (2019).

\section{METODOLOGIA}

A pesquisa caracteriza-se por ser do tipo exploratória e descritiva de abordagem mista com delineamento documental. O ambiente de pesquisa é representado pelo conjunto dos artigos científicos declarados na Plataforma Lattes pelo corpo docente permanente dos programas de pós-graduação stricto sensu do Setor de Ciências Sociais Aplicadas da UFPR. A ocorrência do foco de aplicação desta pesquisa residir na produção docente na pós-graduação justifica-se devido a este nível educacional apresentar maior ocorrência de pesquisas de desenvolvimento científico e tecnológico.

A população da pesquisa considerou os 94 docentes do quadro funcional permanente dos departamentos que compõem os programas de pós-graduação stricto sensu do Setor de Ciências Sociais Aplicadas da UFPR, a saber: Departamento de Administração Geral e Aplicada, Departamento de Contabilidade, Departamento de Economia e Departamento de Ciência e Gestão da Informação. A amostra foi do tipo não-probabilística por julgamento.

\section{Coleta e análise dos dados}

A pesquisa coletou dados de quatro fontes:

a) Sítio do Setor de Ciências Sociais Aplicadas da UFPR, com a finalidade de identificar os docentes do quadro funcional permanente dos programas de pós-graduação stricto sensu;

b) Plataforma Lattes, para levantamento do currículo acadêmico dos docentes e descarregamento do XML dos currículos;

c) Pró-reitoria de Gestão de Pessoas da UFPR (PROGEPE), banco de dados funcional para verificar a afiliação institucional dos docentes (dados tabelados);

d) Banco de dados do resolvedor DOI (dx.doi.org) para confirmar validação do identificador persistente; e

e) Bancos de dados dos diretórios de publicações em acesso aberto DOAJ, Diadorim, Dulcinea e SHERPA/RoMEO para verificar e confirmar a classificação do artigo científico (se acesso aberto).

A análise dos dados foi realizada com a aplicação de um algoritmo de coleta e classificação de artigos científicos elaborado por Ferreira e Sunye (2017, p. 361). A função do algoritmo é identificar quais artigos científicos possuem licença de direito autoral do tipo aberta e que permite o autoarquivamento dos artigos em repositórios digitais (Ferreira, 2016).

O algoritmo possui sete estágios $(0,-1,1,2,3,4,5)$ que executam de forma encadeada a pesquisa dos artigos científicos referenciados pelos docentes na Plataforma Lattes a partir do DOI declarado nas publicações, conforme Figura 1. 


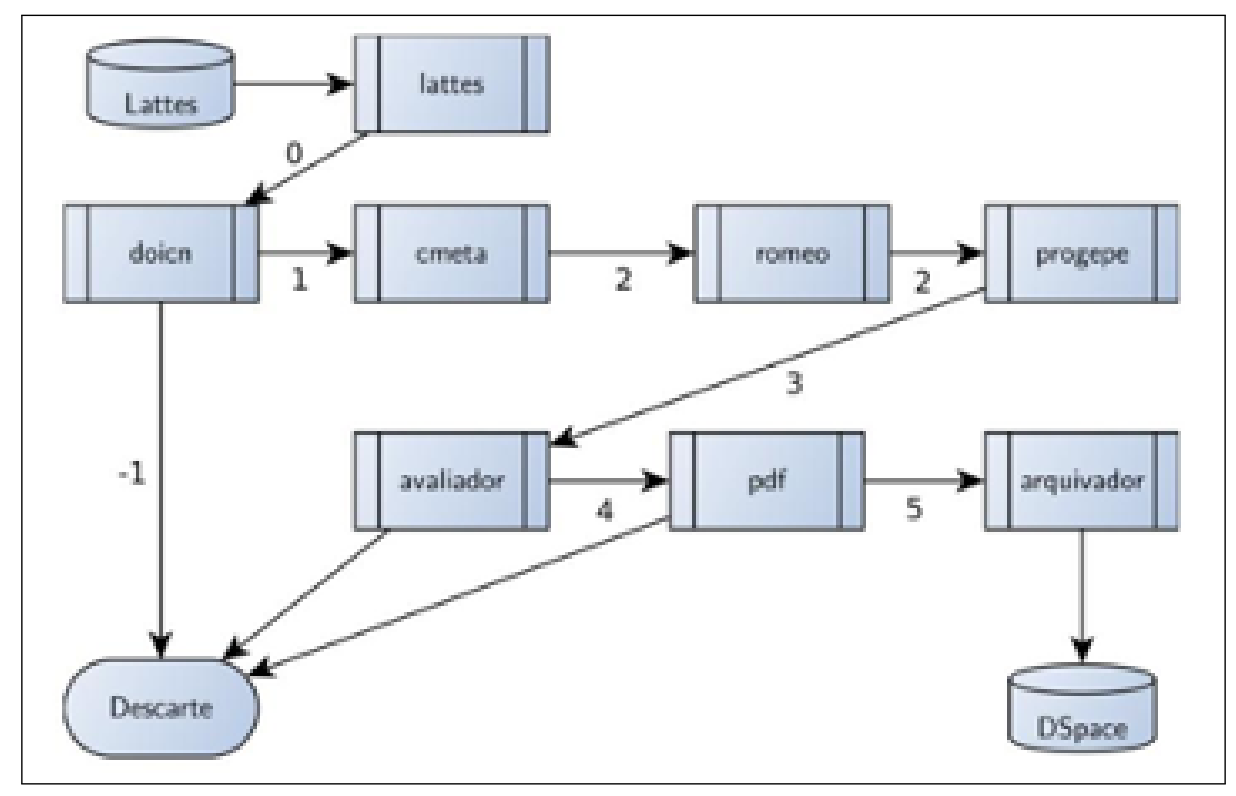

Figura 1. Fluxo de dados para a seleção de artigos científicos.

Fonte: Ferreira (2016); Ferreira e Sunye (2017).

No estágio 0, o componente analisa os metadados do XML dos currículos na Plataforma Lattes identificando os artigos científicos e extraindo o DOI informado para cada referência (Figura 2). Caso o DOI seja válido, o componente armazena os dados em banco de dados relacional (fim do estágio 0). Caso o DOI não seja válido, os dados são sinalizados para descarte (estágio -1).

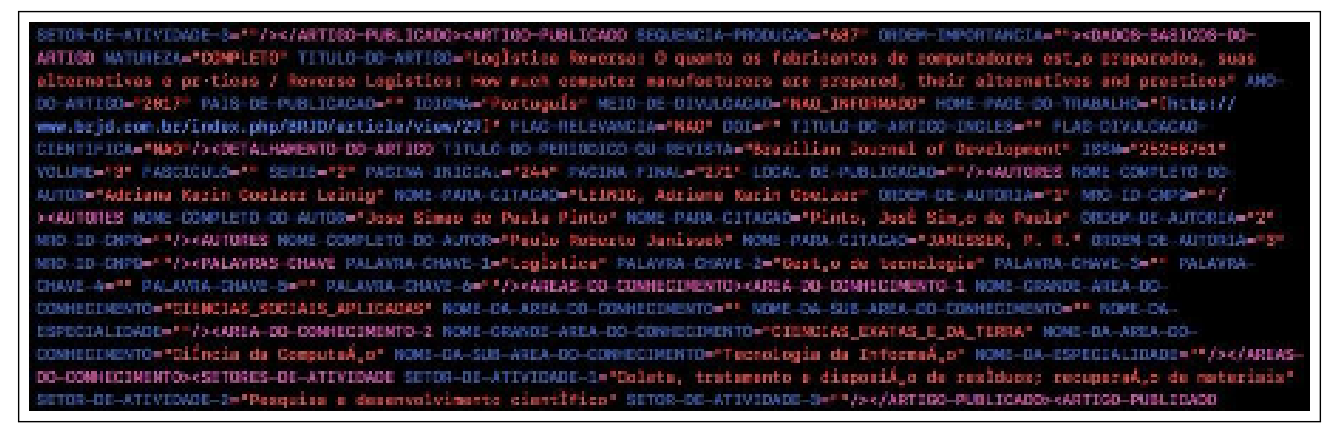

Figura 2. Exemplo de extração de dados XML a partir do Lattes.

Fonte: Dados da pesquisa (2019).

Os metadados consolidados do Lattes e DOI já validados são marcados e armazenados em tabela no banco de dados relacional (estágio 1) e seguem para o estágio 2 no qual é verificado o tipo de licença de direito autoral do artigo. Neste estágio, o componente acessa o banco de dados SHERPA/RoMEO para verificar nos metadados (tags preprints, postprints e pdfversion) o International Standard Serial Number (ISSN) do periódico onde o artigo foi publicado e, assim, definir qual é o tipo de licença atribuída a ele.

Ainda no estágio 2 é realizada a verificação dos nomes dos autores em base de dados funcional (PROGEPE) para atestar que a produção a ser analisada ocorreu durante a estadia do docente na universidade. A verificação ocorre pela data de permanência do docente na instituição. Caso a produção ocorra fora dos períodos de admissão e saída, o artigo é desconsiderado e segue para descarte (estágio -1).

No estágio 3 ocorre a consolidação do vínculo institucional e classificação dos artigos em acesso aberto. O estágio 3 avalia três critérios sobre o artigo, respectivamente: a) os metadados incluem ao menos um ISSN; b) ao menos um dos autores do artigo foi vinculado à instituição e o artigo publicado durante a sua permanência; e c) a versão publicada do artigo, além de estar em acesso aberto, pode ser arquivada em outras bases de dados. Realizada a avaliação, os artigos passíveis de serem arquivados foram atualizados e colocados no próximo estágio. O estágio 4 realizou a coleta do PDF do artigo utilizando um extrator para varrer o código HTML da URL na qual o objeto digital estava armazenado. O conjunto dos metadados e PDF foi marcado e armazenado no banco de dados. No estágio 5, foi criada uma estrutura de diretórios no formato Simple Archive Format para importação e armazenamento em repositório digital (software DSpace).

A utilização do algoritmo elaborado por Ferreira e Sunye (2017) neste trabalho deu-se somente até o estágio 3, pois o objetivo geral da pesquisa é descobrir qual é a forma de publicação adotada pelos docentes dos programas 
de pós-graduação e não a realização da carga de publicações no repositório digital institucional de forma efetiva.

\section{ANÁLISE DOS DADOS E DISCUSSÃO DOS RESULTADOS}

A coleta de dados teve duas fases. A primeira fase iniciou com a identificação dos 93 docentes do quadro funcional permanente dos programas de pós-graduação stricto sensu do Setor de Ciências Sociais da Universidade Federal do Paraná por meio do seu sítio eletrônico. A partir desta identificação, procedeu-se o levantamento e descarga do currículo acadêmico dos docentes da Plataforma Lattes. Dos 93 docentes identificados, apenas dois não tinham currículos cadastrados e por isso foram excluídos da amostra.

A segunda fase da coleta de dados ocorreu com a alimentação do algoritmo de Ferreira e Sunye (2017) com os arquivos XML extraídos da Plataforma Lattes dos 91 docentes, obtendo como resultado 2.672 artigos científicos e 473 DOI únicos e válidos. A diferença entre a quantidade de DOI encontrados e a quantidade total de artigos científicos é explicada devido à possibilidade de um artigo ter múltipla autoria, explicação comprovada pela observação das colaborações entre os docentes, conforme Figura 3.

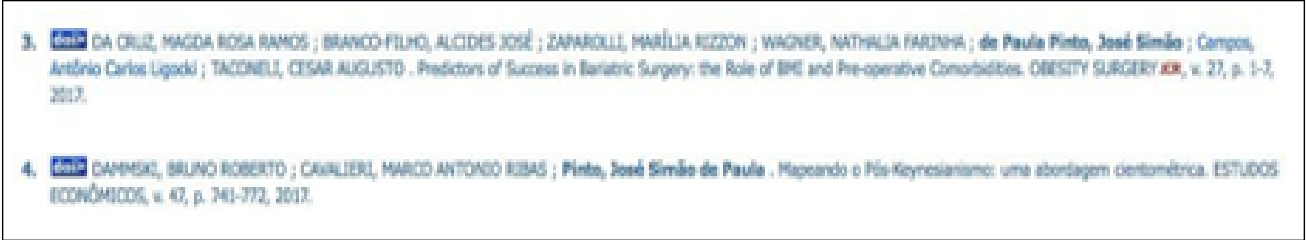

Figura 3. Exemplo de artigo científico de múltipla autoria. Fonte: Dados da pesquisa (2019).

Dos 473 DOIs atribuídos, apenas 260 artigos científicos foram classificados pelo SHERPA/RoMEO como publicados em periódicos de acesso aberto. Destes, 257 artigos foram produzidos durante a permanência do docente na instituição, conforme comparação com o banco de dados funcional da instituição.

A adoção do DOI como critério de seleção para análise do tipo de sistema de publicação adotado pelos docentes ocorre por esta aplicação ser capaz de identificar o conjunto dos metadados e o objeto digital a ele relacionado, independentemente do local de armazenamento ou formato do objeto digital (Sayão, 2007), e até mesmo independentemente do tipo de sistema de publicação. Tanto as grandes editoras e agregadoras de conteúdo acadêmico quanto os portais institucionais de periódicos (instituições públicas ou privadas de pesquisa ou ensino superior) buscam realizar a atribuição do DOI em suas publicações como garantia de disponibilidade de acesso ao conteúdo ou de sua identificação por meio dos metadados.

Os artigos científicos que não recebem identificadores persistentes como o DOI ou outros estão mais propensos a falhas de recuperação e acesso, pois uma mera alteração em diretórios ou sistemas pode levar à alteração da URL e consequente falha no endereço virtual do documento.

De forma parcial, os resultados indicam que parte significativa dos periódicos em que os docentes publicam não realizam atribuição de DOI nos artigos científicos. Ou ainda que não ocorreu a alimentação deste dado em campo específico do formulário de declaração da publicação na Plataforma Lattes. É importante observar que o DOI é uma aplicação paga, e que essa pode ser uma das razões pelas quais não há um número relativamente significativo de atribuições se considerado a quantidade (2.672) total de artigos científicos publicados pelos docentes.

Segundo o OpenDOAJ, o Brasil apresenta o volume de 526.324 artigos científicos publicados em acesso aberto. Em comparação com os Estados Unidos, país com o maior número de publicações científicas (Guimarães, 2018), cuja publicação de artigos em acesso aberto é de 343.707, o cenário brasileiro é significativamente superior ao norte-americano, numa abordagem que considera apenas o volume de artigos em acesso aberto, não considerando as condições operacionais de pesquisa entre os países. A mesma situação ocorre com relação aos periódicos em acesso aberto: Brasil com 1.277 títulos e Estados Unidos com 633 títulos.

Os dados acima não permitem compreender o comportamento de publicação científica dos pesquisadores entre os dois países mencionados e nem no panorama brasileiro. No entanto, fica evidente o destaque brasileiro quanto ao volume de publicações em acesso aberto. Comparando os dados de publicação cadastrados no OpenDOAJ com a autoria dos softwares open source para publicação científica (DSpace, OJS, Fedora, Eprints, Koha, Greenstone ARNO, CDS, entre outros), é possível destacar que o Brasil faz efetivo uso desses sistemas consolidados.

Embora seja latente a força dos movimentos de acesso aberto no âmbito internacional ou nacional e seja vasta e crescente a oferta de ferramentas tecnológicas em open source, observa-se que no recorte institucional do Setor de Ciências Sociais Aplicadas, ainda são poucas as publicações em acesso aberto se comparadas ao total de artigos declarados na Plataforma Lattes. 


\section{CONCLUSÃO}

O objetivo geral desta pesquisa foi responder qual seria a forma de publicação utilizada pelos docentes do Setor de Ciências Sociais Aplicadas da UFPR na comunicação dos seus resultados de pesquisa por meio da análise dos artigos científicos na Plataforma Lattes, classificando a prevalência da forma aberta ou restrita. O resultado foi que dos 473 artigos científicos com DOI, publicados por 91 docentes, 257 destes foram publicados em acesso aberto (open access) e 216 artigos em acesso restrito. O que significa dizer que a maioria dos docentes do quadro permanente publicam suas pesquisas em periódicos de acesso aberto (54\%).

Assim, os dados indicam que a via dourada é a forma de publicação utilizada pela ampla maioria dos docentes do quadro permanente do Setor de Ciências Sociais Aplicadas da UFPR. A partir dos resultados da pesquisa não é possível concluir a razão $46 \%$ dos artigos científicos são publicados em sistema restrito. Entretanto, pode-se apontar como uma das possíveis contribuições a busca constante por reputação por meio dos índices e fatores de impacto e demais métricas em certos momentos mal compreendidos, porém demasiadamente valorizados.

A massiva quantidade de periódicos indexados pelos oligopólios editoriais como Elsevier, Springer, Web of Knowledge, Taylor Francis, EBSCo, entre outros, atuam para promover a comunicação da ciência entre os pares, porém, ao mesmo tempo, o modelo de negócio adotado por essas editoras (sistema de acesso restrito) atrasa a comunicação da ciência entre os pares e seu reflexo na sociedade. Esperava-se que a transição dos periódicos científicos da versão impressa para a versão digital reduzisse o custeio de manutenção das assinaturas de periódicos e bases, o que não ocorreu. O drama vivido pelas bibliotecas desde a década 1980 e 90 (Chimenes, 2016) se mantém nos dias atuais e suscita amplos debates no atual cenário de estagnação e cortes de investimento em pesquisa e pós-graduação no Brasil.

Por outro lado, o resultado de $54 \%$ de artigos científicos publicados em acesso aberto indica um comportamento significativamente positivo na direção dos movimentos de acesso aberto à informação científica. E ainda que existam dúvidas e questionamentos em torno dos critérios de qualidade e confiabilidade das publicações de acesso aberto, bem como a falta de incentivo à publicação aberta por parte de entidades de avaliação da pós-graduação como a CAPES, tornar disponível e acessível a todos a produção científica da universidade é uma das ações do ecossistema da ciência aberta que reaproxima a academia e a ciência da sociedade. Como agenda de trabalhos futuros, um estudo complementar sobre o comportamento do docente/pesquisador poderia auxiliar a compreender suas escolhas para a comunicação dos resultados de pesquisa. 


\section{REFERÊNCIAS}

CAPES. (2018). Distribuição de docentes no brasil por estado. Recuperado de https://geocapes.capes.gov.br/ geocapes/.

Chimenes, A. P. A. (2016). O mercado de publicação científica: um trade off entre difusão e reputação. (Dissertação de mestrado, Universidade Federal do Paraná (UFPR), Curitiba). Recuperado de http://hdl.handle.net/1884/43616.

Costa, S. M. S. (2006). Filosofia aberta, modelos de negócios e agências de fomento: elementos essenciais a uma discussão sobre o acesso aberto à informação científica. Ciência da Informação, 35 (2), 39-50. Recuperado de http://www.scielo .br/pdf/ci/v35n2/a05v35n2.pdf.

Ferreira, E. (2016). Um método de coleta e classificação de metadados de produção científica em repositórios digitais institucionais. (Dissertação de mestrado, Universidade Federal do Paraná (UFPR), Curitiba). Recuperado de http://hdl.handle.net/1884/44782.

Ferreira, E., \& Sunye, M. S. (2017). A method for gathering and classification of scientific production metadata digital libraries. In Proceedings of the 19th international conference on enterprise information systems (iceis 2017) (v. 1, p. 357364). Recuperado de http://www.scitepress.org/Papers/ 2017/63582/63582.pdf.

Guimarães, M. (2018). Brasil é o país com maior publicação em acesso aberto. Recuperado em 2020-0926, de https://revistapesquisa.fapesp.br/brasil-e-o-pais-com -mais-publicacao-cientifica-em-acesso-aberto/.

Harnard, S. (2005). Fast-foward on the green road to open access: the case against mixing up green and gold. Ariadne, 42. Recuperado de http://www.ariadne.ac.uk/ issue $42 /$ harnad.

INEP. (2018). Sinopse estatística da educação superior. Recuperado em 2018-04-04, de http://download.inep.gov.br/informacoes_estatisticas/ sinopses_estatisticas/sinopses_educacao_superior/ sinopse_educacao_superior_2016.zip.

Marcondes, C. H., \& Sayão, L. F. (2001). Integração e interoperabilidade no acesso a recursos informacionais eletrônicos em c\&t: a proposta da biblioteca digital brasileira. Ciência da Informação, 30 (3), 24-33. Recuperado de http://revista.ibict.br/ciinf/article/view/909/946.

Moura, B. R. (2014). O que é a natureza da ciência e qual sua relação com a história e filosofia da ciência? Revista Brasileira de História da Ciência, 7(1), 32-46. Recuperado de https://www.sbhc.org.br/revistahistoria/ view?ID_REVISTA_HISTORIA $=51$.

Mueller, S. P. M. (2006). A comunicação científica e o movimento de acesso aberto ao conhecimento. Ciência da Informação, 35(2), 27-38. Recuperado de http://revista .ibict.br/ciinf/article/view/1138/1293.

Open Archives Initiative. (2007). Open archives initiative organization. Recuperado de https://www.openarchives.org/ organization/.

Sayão, L. F. (2007). Interoperabilidade das bibliotecas digitais: o papel dos sistemas de identificadores persistentes urn, purl, doi, handle system, crossref e openurl. Transinformação, 9(1), 65-82. Recuperado de http:// www.scielo.br/scielo.php?script =sci_abstract\&pid=S0103 $-37862007000100006 \& \operatorname{lng}=$ pt\&nrm $=$ iso\&tlng $=$ pt.

Setenareski, L. E. (2013). Repositórios digitais abertos: um movimento do livre acesso alternativo à estrutura oligo- polizada das editoras científicas (Dissertação de mestrado, Universidade Federal do Paraná (UFPR), Curitiba). Recuperado de http://hdl.handle.net/1884/34734.

Silva, T. E. d., \& Alcará, A. R. (2008). Políticas de acesso aberto à informação científica: iniciativas governamentais. In Anais do ix encontro nacional de pesquisa em ciência da informação. São Paulo. Recuperado de http://enancib.ibict.br/index.php/enancib/ixenancib/ paper/viewFile/3076/2202.

Stallman, R. (2004). Software libre para una sociedad libre. Recuperado de http://biblioweb.sindominio.net/ pensamiento/softlibre/softlibre.pdf.

Universidade Federal do Paraná. (2018). Setor de ciências sociais aplicadas. Recuperado em 2019-01-06, de http:// www.sociaisaplicadas.ufpr.br/portal/departamentos/.

Weitzel, S. R. (2006). O papel dos repositórios institucionais e temáticos na estrutura da produção científica. Em Questão, 12(1), 51-71. Recuperado de https://seer.ufrgs.br/ EmQuestao/article/view/19.
Lima, K. C. R. de, Mendes Junior, R. \& Pinto, J. S. de P. (2019). Artigos científicos publicados em acesso aberto pelos docentes dos programas de pósgraduação stricto sensu no Setor de Ciências Sociais Aplicadas da UFPR. AtoZ: novas práticas em informação e conhecimento, 8(2), 106 - 113. Recuperado de: http://dx.doi.org/10.5380/atoz.v8i2.76295 